\title{
A model of radiating black hole in noncommutative geometry
}

\author{
Piero Nicolini \\ Dipartimento di Matematica e Informatica, Università di Trieste \\ Institut Jožef Stefan, Ljubljana \\ Dipartimento di Matematica, Politecnico di Torino, Turin \\ Istituto Nazionale di Fisica Nucleare, Sezione di Trieste
}

July 26, 2018

\begin{abstract}
The phenomenology of a radiating Schwarzschild black hole is analyzed in a noncommutative spacetime. It is shown that noncommutativity does not depend on the intensity of the curvature. Thus we legitimately introduce noncommutativity in the weak field limit by a coordinate coherent state approach. The new interesting results are the following: i) the existence of a minimal non-zero mass to which black hole can shrink; ii) a finite maximum temperature that the black hole can reach before cooling down to absolute zero; iii) the absence of any curvature singularity. The proposed scenario offers a possible solution to conventional difficulties when describing terminal phase of black hole evaporation.
\end{abstract}

In 1975 Hawking showed that a black hole is able to emit radiation and thus to evaporate 1]. This is one of most intriguing phenomenon in the theory of gravitation, which, after 30 years still remains under debate in particular for what concerns the mysterious explosive end of radiating black holes ( see 2 for a recent review with an extensive reference list ). The black hole phenomenology is part of a larger research area, whose final goal is the formulation of a full quantum theory of gravity. In spite of the promising results that string theory has had in quantizing gravity, the actual calculations of the Hawking radiation are currently obtained by means of quantum field theory in curved space [3]. In fact the black hole evaporation occurs in a semiclassical regime, namely when the density of gravitons is lower than that of the matter field quanta. In spite of this achievements the divergent behavior of the black hole temperature in the final stage of the evaporation remains rather obscure. Indeed in this extreme regime stringy effects cannot be neglected. Recently an improved version of field theory on a noncommutative space time manifold has been proposed as a cheaper way to reproduce the stringy phenomenology, at least in the low energy 
limit. Noncommutativity is encoded in the commutator

$$
\left[\mathbf{x}^{\mu}, \mathbf{x}^{\nu}\right]=i \theta^{\mu \nu} .
$$

where $\theta^{\mu \nu}$ is an anti-symmetric matrix which determines a fundamental cell discretization of spacetime much in the same way as the Planck constant $\hbar$ discretizes the phase space. The basic motivation for assuming (11) consists in an attempt of curing the bad short distance behavior of pointlike sources in ordinary field theory [4, a problem that is only partially solved even in the context of string theory. Indeed preliminary results in a $2 D$ noncommutative spacetime suggest further investigation in this field [5].

The purpose of the paper is to understand whether noncommutativity is able to cure the pathologies which occur in the theory of gravitation, such as the divergent behavior of the black hole temperature during the final stage of the collapse and the curvature singularity at the black hole center. The most direct way to reach this goal is to employ the linearized gravitational field equations as a temporary laboratory to test the effect of noncommutativity until the complete Einstein field equation will be analyzed. There are three good reasons for doing this. First, noncommutativity is an intrinsic property of the manifold and does not depend on the curvature. Indeed there are many examples of noncommutative field theory in flat space, namely in absence of gravity. Thus if any effect is produced by noncommutativity it must appear also in the weak field regime. Second, the concept of "weak" or "strong" field makes sense only if one compares the field strength with a proper scale. In the theory of gravitation we are given of a natural and unique scale, that is the Planck scale. Thus with respect to the Planck scale the gravitational field strength can still be considered "weak" even in the vicinity of a black hole, justifying the adoption of linearized field equations until the horizon radius is larger than the Planck length. Third, we will show that, in the considered case of Schwarzschild geometry, the expression of the temperature does not depend on the weak field expansion.

In this spirit we assume as infinitesimal dimensionless parameter

$$
\frac{\phi_{N}}{\phi_{P l} .} \ll 1
$$

where $\phi_{N}$ is the Newtonian potential, while $\phi_{P l} .=M_{P l} / l_{P l}$. As a peculiar aspects of Schwarzschild space time we observe that $g_{r r}$ is the only component of the metric tensor that is affected by linearization. Indeed the line element reads

$$
d s^{2}=-\left(1+\frac{2 \phi_{N}}{\phi_{P l .}}\right) d t^{2}+d l^{2}
$$

where $d l^{2}$ is the Euclidean spatial line element. The Hawking temperature is given by definition

$$
T_{H} \equiv-\left(\frac{1}{4 \pi \sqrt{-g_{00} g_{11}}} \frac{d g_{00}}{d r}\right)_{r=r_{H}} .
$$


As anticipated one finds the temperature $T_{H}=1 / 8 \pi M$, that is coincident with the expression given by the exact theory, since $T_{H}$ essentially depends on $g_{00}$ only.

There are many approaches to implement noncommutativity in a field theory. The underlying philosophy of these approaches is to modify the distribution of point like sources in favor of smeared objects. Such prescription is in agreement with the conventional procedure for the regularization of UV divergences by the introduction of a cut off. In recent papers [6, 7] the coordinate coherent state approach has been proposed and the precise distribution of field sources has been determined to be the Gaussian distribution. The resulting field theory is UV finite while Lorentz invariance and unitariety are preserved 8. In order to incorporate noncommutativity one can observe that there exists a unique linearized Einstein equation

$$
\vec{\nabla}^{2} \phi_{N}=4 \pi G_{N} \delta(r)
$$

namely the classical Poisson equation for a point-like source described by Dirac delta-function, with $M$ the mass of the source and $G_{N}$ the Newton constant. At this point we stress that noncommutativity is an intrinsic property of the manifold, the contrary of curvature, that is a geometrical tool defined over the underlying manifold to measure the strength of the gravitational field. For these reasons curvature and noncommutativity are independent concepts. Thus, noncommutative modification of Schwarzschild space time, once introduced at a given curvature, will remain valid in any other field strength regime. Our way of reasoning is in perfect agreement with the standard procedure to implement noncommutativity in quantum field theory where the strength of the field is not an issue [6], 7. The physical effect of noncommutativity is that the very concept of point-like object is no more meaningful and one has to deal with smeared objects only. To practical purpose the implementation of noncommutativity by means of coherent state approach is realized by substituting the position Diracdelta, characterizing point-like structures, with Gaussian function of minimal width $\sqrt{\theta}$ describing the corresponding smeared structures. At this point the noncommutative field equation reads 9

$$
\begin{aligned}
\vec{\nabla}^{2} \phi_{N} & =4 \pi G_{N} \rho_{\theta}(\vec{x}) \\
\rho_{\theta}(\vec{x}) & =\frac{M}{(2 \pi \theta)^{3 / 2}} \exp \left(-\vec{x}^{2} / 4 \theta\right)
\end{aligned}
$$

where $\rho_{\theta}$ is the Gaussian mass density. We obtain the NC version of linearized Schwarzschild line element as

$$
d s^{2}=-\left(1+\frac{2 \gamma \phi_{N}}{\sqrt{\pi} \phi_{P l}}\right) d t^{2}+d l^{2}
$$

where $\gamma$ is the lower incomplete Gamma function, with the definition

$$
\gamma \equiv \gamma\left(1 / 2, r^{2} / 4 \theta\right) \equiv \int_{0}^{r^{2} / 4 \theta} d t t^{-1 / 2} e^{-t}
$$


In the commutative limit $r / 2 \sqrt{\theta} \rightarrow \infty$ the standard linearized Schwarzschild metric is reproduced. The line element (7) describes the geometry of a noncommutative black hole under the condition that $r_{H} \gg l_{P l}$. and should give us useful indications about possible noncommutative effects on the Hawking radiation.

To calculate the Hawking temperature we need the event horizon radius $r_{H}$, that is defined by the vanishing of "unperturbed" $g_{00}$. In our case it leads to the implicit equation ${ }^{1}$

$$
r_{H}=\frac{2 M}{\sqrt{\pi}} \gamma\left(1 / 2, r_{H}^{2} / 4 \theta\right)
$$

Rewriting (9) in terms of the upper incomplete Gamma function as

$$
r_{H}=2 M\left[1-\frac{1}{\sqrt{\pi}} \Gamma\left(1 / 2, r_{H}^{2} / 4 \theta\right)\right]
$$

one recovers the conventional Schwarzschild radius plus $\theta$-corrections. In the "large radius" regime $r_{H}^{2} / 4 \theta>>1$ equation (10) can be solved by iteration. At the first order approximation, we find

$$
r_{H}=2 M\left(1-\sqrt{\frac{\theta}{\pi}} \frac{1}{M} e^{-M^{2} / \theta}\right)
$$

The effect of noncommutativity is exponentially small, which is reasonable to expect since for large radii with respect to $\sqrt{\theta}$, spacetime can be considered as a smooth classical manifold (commutative limit). On the other hand, in the opposite limit, one expects significant changes due to the spacetime fuzziness. To this purpose it is convenient to invert (10) and consider the black hole mass $M$ as function of $r_{H}$

$$
M\left(r_{H}\right)=\frac{r_{H} \sqrt{\pi}}{2 \gamma\left(1 / 2, r_{H}^{2} / 4 \theta\right)}
$$

In such a limit, i.e. $r_{H} / \sqrt{\theta} \ll 1$, the equation (12) leads to

$$
M \rightarrow M_{0}=0.5 \sqrt{\pi \theta}
$$

which is a new and interesting result. Noncommutativity implies a minimal non-zero mass that allows the existence of an event horizon (see Figure 1). If the starting black hole mass is such that $M>M_{0}$, it can radiate through the Hawking process until the value $M_{0}$ is reached. At this point the horizon has totally evaporated leaving behind a massive relic. Black holes with mass $M<M_{0}$ do not exist. An equivalent scenario arises from the behavior of $g_{00}$ (see Figure 2). There are three pictures

1. for $M>M_{0}$ (dotted curve) there is a black hole with regular metric in the origin;

2. for $M=M_{0}$ (solid curve) the event horizon is shrank to the origin. 


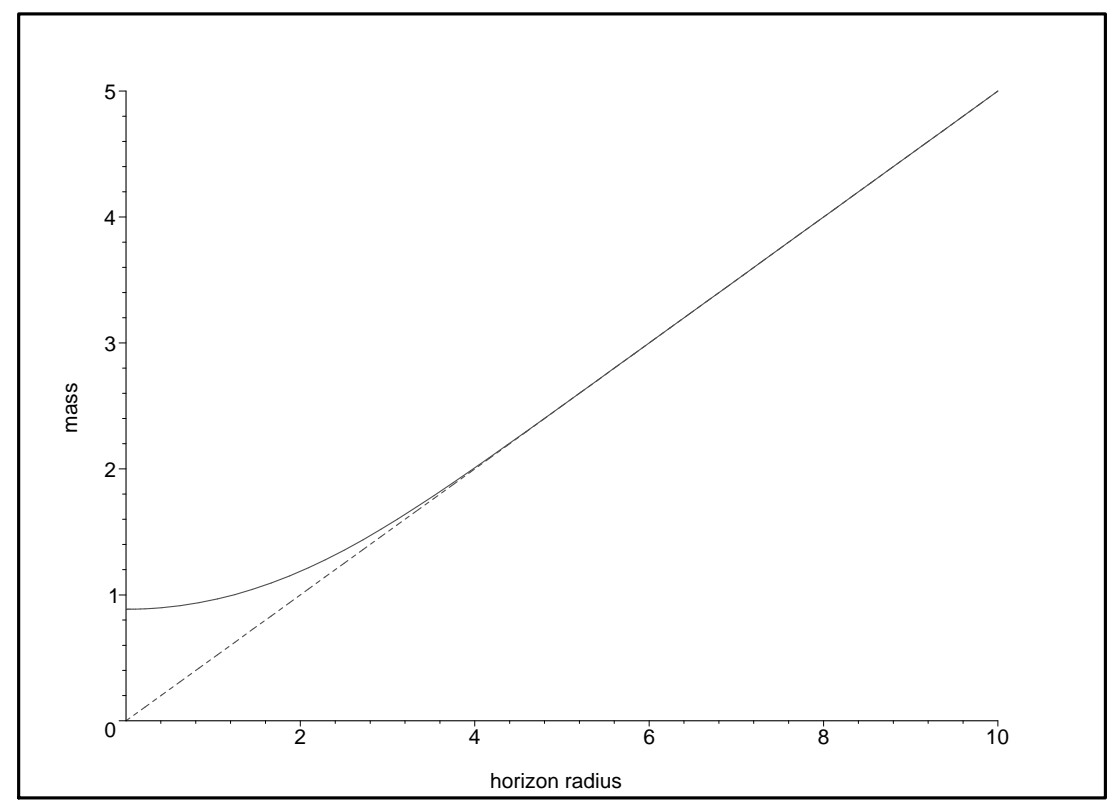

Figure 1: Mass vs horizon relation. In the commutative case, dashed line, the mass is the linear function $M=r_{H} / 2$ vanishing at the origin, while in the noncommutative case, solid line, $M\left(r_{H} \rightarrow 0\right)=M_{0}$, i.e. for $M<M_{0}$ there is no event horizon.

3. for $M<M_{0}$ (dashed curve) there is no horizon.

To understand the physical nature of the mass $M_{0}$ remnant, let us also consider the black hole temperature as a function of $r_{H}$. It is given by

$$
T_{H}\left(r_{H}\right)=\frac{1}{4 \pi}\left[\frac{1}{r_{H}}-\frac{\gamma^{\prime}\left(1 / 2 ; r_{H}^{2} / 4 \theta\right)}{\gamma\left(1 / 2 ; r_{H}^{2} / 4 \theta\right)}\right]
$$

where the "prime" denotes differentiation with respect to $r$. In the large radii limit, i.e. $r_{H}^{2} / 4 \theta>>1$, one recovers the standard result for the Hawking temperature

$$
T_{H}=\frac{1}{4 \pi r_{H}}
$$

while noncommutativity becomes crucial, when $r_{H} \sim \sqrt{\theta}$. In the conventional ( commutative ) case $T_{H}$ diverges and this puts limit on the validity of the conventional description of Hawking radiation. Against this scenario, formula (14) leads to

$$
T_{H} \sim \frac{r_{H}}{24 \pi \theta}, \quad \text { as } \quad \frac{r_{H}}{\sqrt{\theta}} \rightarrow 0
$$

\footnotetext{
${ }^{1}$ We use convenient units $G_{N}=1, c=1$.
} 


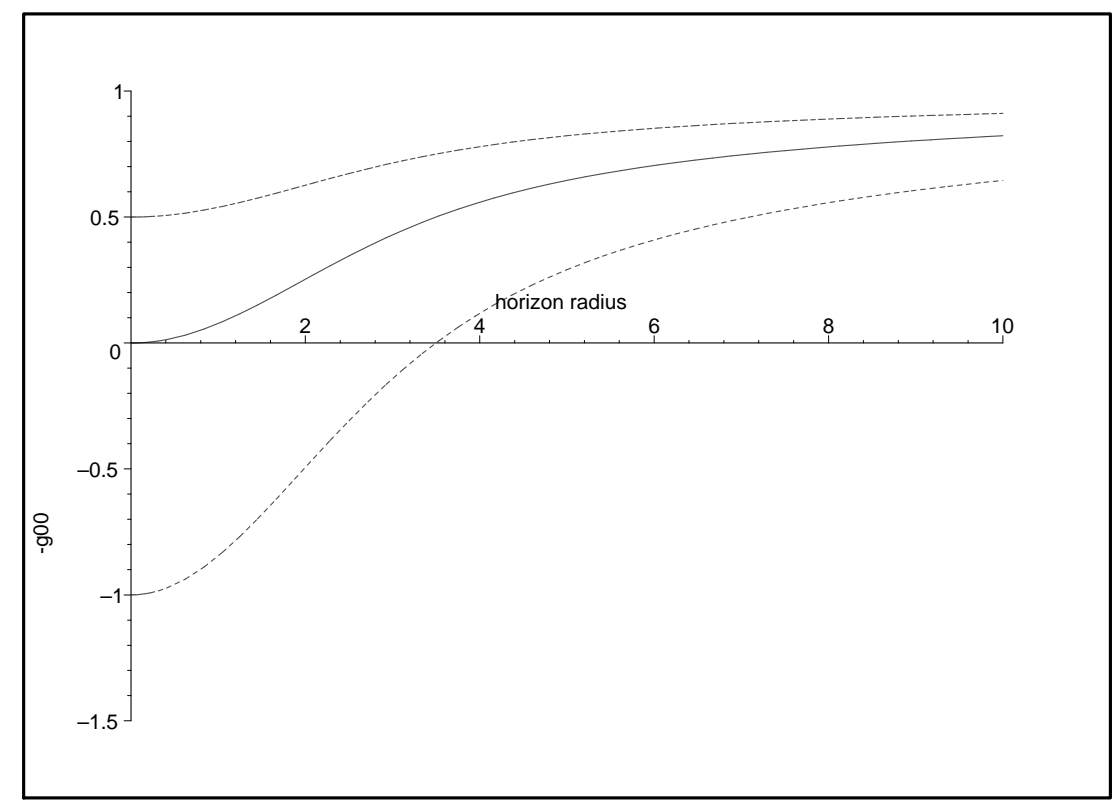

Figure 2: The function $-g_{00}$ vs the radial distance $r$ for some value of the mass $M$. The dashed line, corresponds to a mass $M=0.5 M_{0}$ for which there is no event horizon. The dotted line corresponds to a mass $M=2 M_{0}$, which describes a black hole, which is regular at its center $r=0$. The solid curve is the borderline case, namely the case of $M=M_{0}$ in which the horizon radius $r_{H}$ is shrank in the origin.

This is another intriguing result that has two important consequences. Firstly, the emerging picture is that the black hole has reached zero temperature and the horizon has completely evaporated. Nevertheless, we are left with a frozen, massive, remnant. Secondly, passing from the regime of large radius to the regime of small radius, (15) and (16), implies the existence of a maximum temperature which is confirmed by the plot in Figure 3. The plot gives the value $T_{H}^{M a x} .=2.18 \times 10^{-2} / \sqrt{\theta}$. The temperature behavior shows that noncommutativity plays the same role in General Relativity as in Quantum Field Theory, i.e. removes short distance divergences. The resulting picture of black hole behavior goes as follows. For $M>>M_{0}$ the temperature is given by (15) up to exponentially small corrections, and it increases, as the mass is radiated away. $T_{H}$ reaches a maximum value at $r_{H}=2.74 \sqrt{\theta}$, and then decreases down to zero as $r_{H}$ goes to zero.

At this point, important issue of Hawking radiation back-reaction should be discussed. In commutative case one expects relevant back-reaction effects during the terminal stage of evaporation because of huge increase of temperature [10]. In our case, the role of noncommutativity is to cool down the black hole in the 


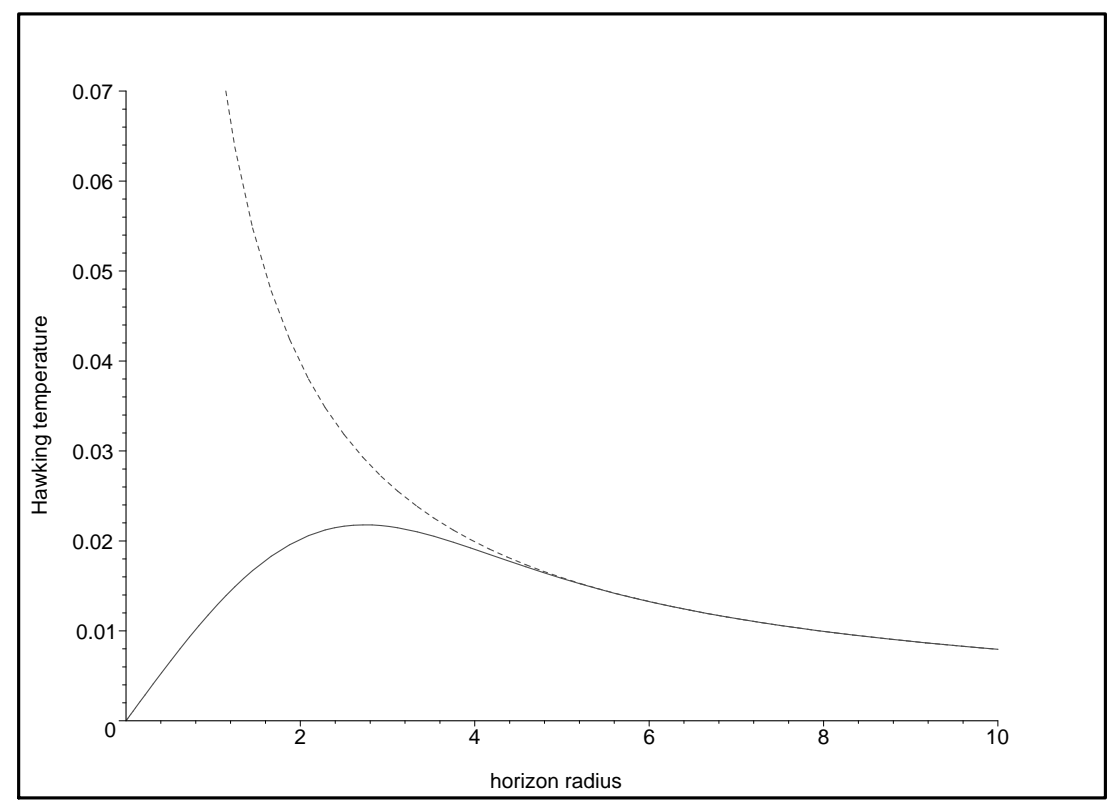

Figure 3: Hawking temperature $T_{H}$ as a function of the horizon radius $r_{H}$. In the noncommutative case, solid curve, one see the temperature reaches a maximum value $T_{H}^{M a x} .=2.18 \times 10^{-2} / \sqrt{\theta}$ for $r_{H}=2.74 \sqrt{\theta}$, and then decreases to zero as $r_{H} \rightarrow 0$. The commutative, divergent behavior, dashed curve, is cured.

final stage. As a consequence, there is a suppression of quantum back-reaction since the black hole emits less and less energy. Eventually, back-reaction may be important during the maximum temperature phase. In order to estimate its importance in this region, let us look at the thermal energy $E=T$ and the total mass $M$ near $r_{H}=2.74 \sqrt{\theta}$. From (12) one finds $M \sim \sqrt{\theta} M_{P l \text {. }}^{2}$. In order to have significant back-reaction effect $T_{H}^{M a x}$ should be of the same order of magnitude as $M$. This condition leads to the estimate

$$
\sqrt{\theta} \sim 10^{-1} l_{P l .} \sim 10^{-34} \mathrm{~cm}
$$

Expected values of $\sqrt{\theta}$ are above the Planck length $l_{P l}$. and (17) indicates that back-reaction effects are suppressed even at $T_{H}^{M a x} \approx 10^{18} \mathrm{GeV}$. For this reason we can safely use unmodified form of the metric (7) during all the evaporation process.

As it appears, at the final stage of evaporation a mass $M_{0}$ is left behind. One would be tempted to say that the black hole evaporation has produced a naked singularity of mass $M_{0}$. We are going to show that this is not the case. In the linearized geometry the curvature tensors can be written in terms of the Ricci scalar $R$. Thus for the metric (7) the curvature tensors are everywhere 
regular, since the scalar $R$ turns out to be

$$
R=-8 \pi G_{N} \rho_{\theta}
$$

In particular, for what concerns the case of a naked singularity one should

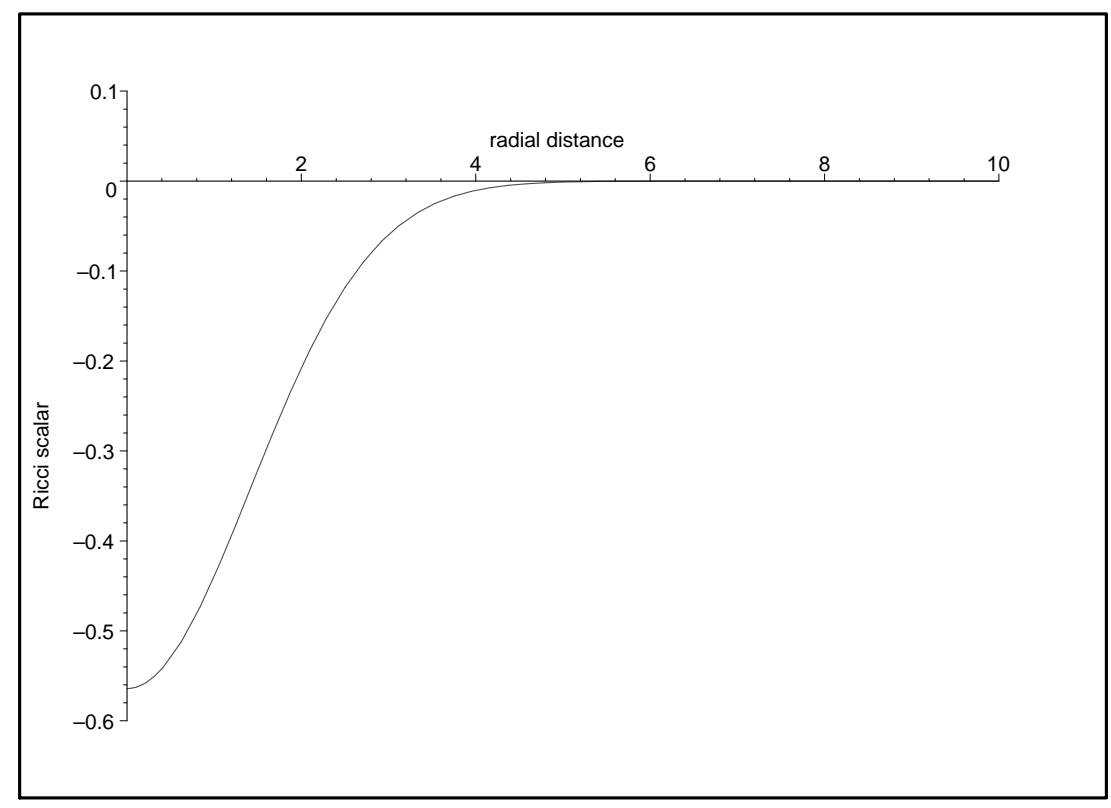

Figure 4: Ricci scalar as function of $r$. The curvature singularity in the origin is removed, and $R(0)=-M / \sqrt{\pi} \theta^{3 / 2}$.

obtain a divergent curvature in the origin, while the short distance behavior of $R$ is

$$
R \simeq-\frac{M}{\sqrt{\pi} \theta^{3 / 2}}
$$

Indeed for $r<<\sqrt{\theta}$ the geometry of the frozen relic has constant and negative curvature. On the other hand, in the commutative limit $r / \sqrt{\theta} \gg 1$, one can check that (18) reproduces the usual Schwarzschild scalar curvature. Indeed the Dirac delta distribution is restored in (18) at large distances.

Regular black holes have been introduced as ad hoc models implementing the idea of a maximum curvature 11. On the other hand we have found here an equivalent non singular black hole as a solution of linearized Einstein equation with a source suitably prescribed by coordinate commutativity.

As a conclusion, the results derived in this work show that the coordinate coherent state approach to noncommutative effects can cure the singularity problems at the terminal stage of black hole evaporation. We have shown that noncommutativity is an intrinsic property of the manifold itself and thus unaffected 
by the distribution of matter. Matter curves a noncommutative manifold in the same way as it curves a commutative one, but cannot produce singular structures. Specifically, we have shown that there is a minimal mass $M_{0}=0.5 \sqrt{\pi \theta}$ to which a black hole can decay through Hawking radiation. The reason why it does not end-up into a naked singularity is due to the finiteness of the curvature at the origin. The everywhere regular geometry and the residual mass $M_{0}$ are both manifestations of the Gaussian de-localization of the source in the noncommutative spacetime. On the thermodynamic side, the same kind of regularization takes place eliminating the divergent behavior of Hawking temperature. As a consequence there is a maximum temperature that the black hole can reach before cooling down to absolute zero. As already anticipated in the introduction, noncommutativity regularizes divergent quantities in the final stage of black hole evaporation in the same way it cured UV infinities in noncommutative quantum field theory. We have also estimated that back-reaction does not modify the original metric in a significant manner.

\section{Acknowledgement}

The author thanks the "Dipartimento di Fisica Teorica dell'Università di Trieste", the PRIN-COFIN project 2004 "Metodi matematici per le teorie cinetiche" and the CNR-NATO program for financial support.

\section{References}

[1] Hawking S W 1975 Comm. Math. Phys. 43199

[2] Padmanabhan T 2005 Phys. Rep. 40649

[3] Balbinot R Fabbri A Nicolini P Sutton P J 2002 Phys. Rev. D 66024014 Balbinot R Fabbri A Frolov V Nicolini P Sutton P J Zelnikov A 2001 Phys. Rev. D 63084029

[4] Nicolini P 2004 Vacuum energy momentum tensor in (2+1) NC scalar field theory arXiv:hep-th/0401204

[5] Nicolini P Smailagic A and Spallucci E 2005 The fate of radiating black holes in noncommutative geometry arXiv:hep-th/0507226

[6] Smailagic A Spallucci E 2003 J. Phys. A 36 L467

[7] Smailagic A Spallucci E 2003 J. Phys. A 36 L517

[8] Smailagic A Spallucci E 2004 J. Phys. A 377169

[9] Gruppuso A 2005 J. Phys. A 382039

[10] Balbinot R Barletta A 1989 Class. Quantum Gravity 6 195; Balbinot R Barletta A Class. Quantum Gravity 6203 
[11] Easson D A 2003 JHEP 0302:037; Hayward S A 2005, "Formation and evaporation of regular black holes" arXiv:gr-qc/0506126 